\title{
Multimodality Therapy of Localized Gastric Adenocarcinoma
}

\author{
Brian Badgwell, MD, MS
}

\begin{abstract}
Surgical resection is the primary method of obtaining a potential cure for patients with gastric adenocarcinoma. However, chemotherapy or chemoradiotherapy offer significant improvement in survival over surgery alone. Much of the difficulty in deciding the optimal treatment strategy is choosing between perioperative chemotherapy or adjuvant chemoradiotherapy. Adding to the complexity is the potential for incorporating treatment strategies based on clinical trials performed in Asia. There is likely a difference in tumor biology between Eastern and Western patients with gastric adenocarcinoma, in addition to the clear differences in screening, pathologic assessment, and surgical technique. Even among tumors in Western populations, heterogeneity of histology, anatomy, and staging among studies make cross-trial comparisons difficult. There is also active controversy surrounding the staging and management of gastroesophageal junction tumors. Lastly, the benefit of extended lymph node dissection is not clear based on the existing studies and has not been firmly established as a surgical standard of care for all patients. Future goals include better classification of gastric cancer, continued pursuit of randomized trials in Western populations, standardization of surgical technique, and incorporation of targeted therapies.
\end{abstract}

J Natl Compr Canc Netw 2016;14(10)1321-1327

\section{NCCN: Continuing Education}

\section{Accreditation Statement}

This activity has been designed to meet the educational needs of physicians and nurses involved in the management of patients with cancer. There is no fee for this article. No commercial support was received for this article. The National Comprehensive Cancer Network (NCCN) is accredited by the ACCME to provide continuing medical education for physicians.

NCCN designates this journal-based CME activity for a maximum of 1.0 AMA PRA Category 1 Credit $^{\mathrm{TM}}$. Physicians should claim only the credit commensurate with the extent of their participation in the activity.

NCCN is accredited as a provider of continuing nursing education by the American Nurses Credentialing Center's Commission on Accreditation.

NCCN designates the education activity for a maximum of 1.0 contact hour. Accreditation as a provider refers to recognition of educational activities only; accredited status does not imply endorsement by NCCN or ANCC of any commercial products discussed/

From The University of Texas MD Anderson Cancer Center, Houston, Texas. Submitted June 13, 2016; accepted for publication September 6, 2016. The author has disclosed that he has no financial interests, arrangements, affiliations, or commercial interests with the manufacturers of any products discussed in this article or their competitors.

Correspondence: Brian Badgwell, MD, MS, The University of Texas MD Anderson Cancer Center, 1400 Pressler Street, Unit 1484, Houston, TX 77030. E-mail: bbadgwell@mdanderson.org

\section{EDITOR}

Kerrin M. Green, MA, Assistant Managing Editor, JNCCN—Journal of the National Comprehensive Cancer Network

Ms. Green has disclosed that she has no relevant financial relationships. displayed in conjunction with the educational activity. Kristina M. Gregory, RN, MSN, OCN, is our nurse planner for this educational activity.

All clinicians completing this activity will be issued a certificate of participation. To participate in this journal CE activity: 1) review the learning objectives and author disclosures; 2 ) study the education content; 3 ) take the posttest with a $66 \%$ minimum passing score and complete the evaluation at http://education.ncon.org/ node/79642; and 4) view/print certificate.

Release date: October 10, 2016; Expiration date: October 10, 2017

\section{Learning Objectives}

Upon completion of this activity, participants will be able to:

- Compare and contrast gastric cancer tumor biology, staging, surgical techniques and incorporation of adjuvant therapy between Eastern and Western clinical trial populations

- Summarize the need for a broad multidisciplinary approach to the management of patients with gastric cancer

\section{CE AUTHORS}

Deborah J. Moonan, RN, BSN, Director, Continuing Education, has disclosed that she has no relevant financial relationships.

Kristina M. Gregory, RN, MSN, OCN, Vice President, Clinical Information Operations, has disclosed that she has no relevant financial relationships. Rashmi Kumar, PhD, Senior Manager, Clinical Content, has disclosed that she has no relevant financial relationships.

Hema Sundar, PhD, Oncology Scientist/Senior Medical Writer, has disclosed that she has no relevant financial relationships. 


\section{Multidisciplinary Approach}

Although multimodality therapy in gastric cancer is primarily focused on surgical, medical, and radiation oncology, a broad multidisciplinary team approach, including conference attendance with radiologists, gastroenterologists, and pathologists, is needed to determine the optimal staging and treatment approach. ${ }^{1}$ This approach facilitates a multidisciplinary discussion that incorporates clinicians who would otherwise not be directly involved, and therefore goes beyond simple referral for multidisciplinary clinical evaluation. This approach is not emphasized in regions of the world where a surgery-first approach is standard of care, radiation therapy is infrequently used, and many cancers are found at an early stage and don't require adjuvant chemotherapy.

Worldwide, gastric cancer is the third leading cause of death in men and the fifth leading cause of death in women. ${ }^{2}$ Gastric cancer incidence rates are highest in Eastern Asia, Central and Eastern Europe, and South America, whereas rates are low in Northern America. One of the first decision points in analyzing the existing studies on gastric cancer and deciding on the optimal treatment approach is whether gastric cancer is the same in Eastern and Western populations. In regard to gastric cancer epidemiology for this review, Eastern studies will be defined as trials from Japan, South Korea, Taiwan, and China. Western populations will be defined as the United States and Western Europe. There are few trials from South America, and further research is needed to determine whether survival for patients from South America is similar to that for Eastern or Western populations.

The difference in incidence rates is partly attributed to differences in food preservation techniques, regional dietary preferences, and Helicobacter pylori infection rates. Regional differences in incidence support variations in tumor biology that raise concern over using Eastern treatment strategies in Western populations. Western patients with gastric cancer are also older and have a higher frequency of several adverse prognostic factors, such as proximal tumor location, poorly differentiated histology, and the presence of signet ring cells.

In addition to the clear differences in tumor biology and histology, there are also differences in the stage at presentation (primarily due to widespread screening in Japan and South Korea), surgical technique, and adjuvant therapy. Because of these differences, it is difficult to compare patients between regions based on stage and to fully adjust for differences in staging and treatment. Investigators have explored this challenging research question, reporting a better survival rate for Eastern than Western patients on adjusted analysis, although there are significant differences even among Eastern countries, and meaningful comparison will likely require more than multivariable statistical analysis. ${ }^{3-5}$ Current NCCN Clinical Practice Guidelines in Oncology (NCCN Guidelines) for Gastric Cancer are primarily based on Western studies, and we will highlight recommendations with regional implications regarding systemic therapy or surgery herein. ${ }^{1}$ Table 1 highlights the differences in the various trials that will be covered in this review, stratified by whether the trial was performed in Eastern or Western populations of patients with gastric cancer.

\section{Staging for Localized Gastric Cancer}

A chest/abdominal/pelvic CT scan is currently the mainstay for imaging for resectable gastric adenocarcinoma. PET/CT imaging is also recommended if clinically indicated in patients without evidence of metastatic disease on routine CT imaging, although this recommendation requires further support. ${ }^{1}$ Gastric adenocarcinoma, particularly diffuse type, signet ring cell type, or tumors with mucinous histology, may not demonstrate increased uptake on PET/CT imaging, and conflicting results have been reported on the association between increased initial standardized uptake value and improved survival in patients receiving preoperative therapy. ${ }^{6-8}$

Diagnostic laparoscopy with cytology is an important consideration for patients with potentially resectable cancer, because approximately $30 \%$ of patients will have radiologically occult carcinomatosis or positive cytology. ${ }^{1,9}$ In addition to the benefit of avoiding laparotomy, patients with low-volume peritoneal disease have the potential for relatively long overall survival and should be considered for trials aimed at improving what has historically been reported as dismal survival. ${ }^{10,11}$

Many patients will present for initial oncologic evaluation with only a diagnostic endoscopy report that is inadequate for multimodality recommendations. Repeat endoscopy with endoscopic ultrasound can be crucial in determining the anatomic location of the tumor and the proximity to the gastroesopha- 
Multimodality Therapy for Gastric Cancer

\begin{tabular}{|c|c|c|c|c|c|}
\hline Trial & & Outcome & Surgery Alone & Chemotherapy & Chemoradiotherapy \\
\hline \multicolumn{6}{|c|}{ Western centers } \\
\hline & Intergroup $0116^{18}$ & $3-y$ OS & $41 \%$ & & $50 \%$ \\
\hline & MAGIC $^{12}$ & $5-y$ OS & $23 \%$ & $36 \%$ & \\
\hline & $\mathrm{FNCLCC}^{13}$ & $5-y$ OS & $24 \%$ & $38 \%$ & \\
\hline & CROSS $^{20}$ & $5-y$ OS & $34 \%$ & & $47 \%$ \\
\hline \multicolumn{6}{|c|}{ Eastern centers } \\
\hline & ACTS-GC 41 & $5-y$ OS & $61 \%$ & $72 \%$ & \\
\hline & CLASSIC $^{16}$ & $3-y$ OS & $78 \%$ & $83 \%$ & \\
\hline & ARTIST $^{17}$ & $5-y$ OS & & $73 \%$ & $75 \%$ \\
\hline
\end{tabular}

Abbreviation: OS, overall survival.

geal junction (GEJ). Although lesions amenable to endoscopic mucosal resection (EMR) or endoscopic submucosal dissection (ESD) are rare in the United States, lesions amenable to such techniques include nodules $2 \mathrm{~cm}$ or less, well- or moderately well-differentiated, without involvement beyond the superficial submucosa, and with clear margins. ${ }^{1}$ These guidelines exclude most cancers diagnosed in the West, and consideration must be given to local expertise in not only the endoscopic resection but also the quality of the ultrasound documenting an early lesion.

\section{Chemotherapy for Localized Gastric Cancer}

\section{Perioperative Chemotherapy}

There are preoperative and postoperative choices for administering chemotherapy to patients with potentially resectable gastric cancer. Preoperative chemotherapy, as part of a planned perioperative approach, is based on the MAGIC trial in which patients received epirubicin, cisplatin, and infusional FU for 3 cycles preoperatively and postoperatively compared with surgery alone. ${ }^{12}$ The 5 -year survival rates were $36 \%$ in the perioperative chemotherapy arm versus $23 \%$ in the surgery alone arm, despite only $42 \%$ of patients randomized to perioperative chemotherapy completing all 6 cycles. There was also variability in surgical technique, with only $40 \%$ of patients having undergone a D2 lymph node dissection. Notably, there was not a significant difference in postoperative complications, providing support to the concept that preoperative therapy does not increase surgical risk. Variability in tumor location was also present, with approximately $25 \%$ of patients having lower esophageal or GEJ tumors.

Further support for perioperative therapy is found in the French FNCLCC/FFCD trial, in which patients with distal esophageal $(n=25)$, gastroesophageal $(n=144)$, and gastric $(n=55)$ adenocarcinoma were randomized to perioperative therapy with 5 -FU and cisplatin versus surgery alone. ${ }^{13}$ Similar to the MAGIC trial, 5-year overall survival was higher in the perioperative therapy group (38\%) compared with the surgery-alone group (24\%). ${ }^{13}$ Another similarity to the MAGIC trial is that only approximately half of the patients were able to receive postoperative chemotherapy, as outlined in Figure 1.

\section{Postoperative Chemotherapy}

Studies of postoperative chemotherapy alone are more conflicting and the optimal regimen is less clear. Because of the number of studies of postoperative

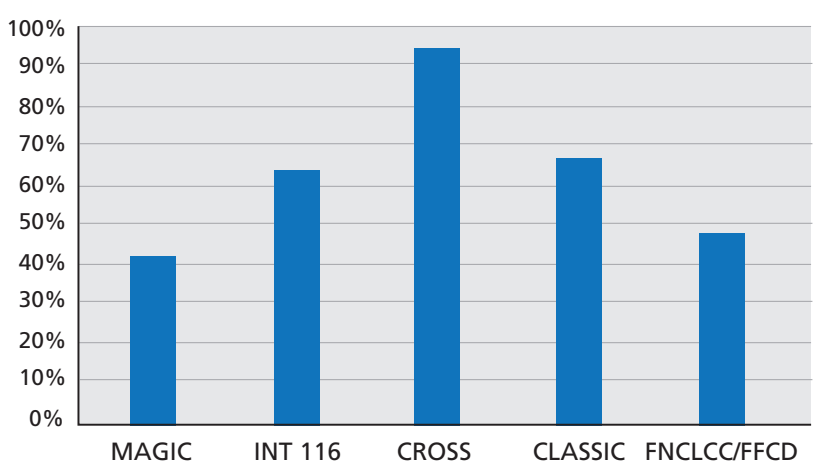

Figure 1. Completion rates for treatment arms in randomized controlled trials of surgery with chemotherapy or chemoradiotherapy versus surgery alone in patients with gastric or gastroesophageal cancer. 
Badgwell

chemotherapy compared with surgery alone in gastric cancer, authors have used meta-analyses to help clarify this area of controversy. Although reviews that included Eastern trials tend to show a benefit for postoperative chemotherapy, studies limited to Western populations suggest less benefit. ${ }^{14,15}$ Current NCCN Guidelines for Gastric Cancer include postoperative chemotherapy (capecitabine with oxaliplatin or cisplatin) for patients who have undergone a D2 lymph node dissection based on the CLASSIC and ARTIST trials. ${ }^{1,16,17}$ In the CLASSIC trial, patients in South Korea, China, and Taiwan who had undergone gastrectomy were then randomized to capecitabine plus oxaliplatin for 8 cycles versus no postoperative therapy. Five-year overall survival was higher in patients who received postoperative chemotherapy (78\% vs 69\%). ${ }^{16}$ The ARTIST trial compared postoperative chemotherapy (capecitabine and cisplatin) versus postoperative chemotherapy followed by chemoradiotherapy (capecitabine with $45 \mathrm{~Gy}$ ); results showed no difference in overall survival. ${ }^{17}$

\section{Chemoradiotherapy for Localized Gastric Cancer}

Similar to chemotherapy, there are also preoperative and postoperative choices for the application of chemoradiotherapy in localized gastric cancer. Current recommendations for postoperative chemoradiotherapy are primarily based on the Intergroup 116 trial, a US study in which patients received one cycle of FU/leucovorin, then FU/leucovorin with 45 Gy, followed by 2 more cycles of FU/leucovorin compared with surgery alone. ${ }^{18}$ Three-year overall survival rates were higher in patients treated with chemoradiotherapy (50\%) compared with surgery alone $(41 \%)$. The most recent analysis continues to demonstrate a survival benefit for adjuvant chemoradiotherapy, with $24 \%$ more patients in the treatment arm disease free compared with the surgery alone arm. ${ }^{19}$ The ARTIST trial from South Korea failed to show a benefit for postoperative chemoradiotherapy compared with chemotherapy; however, subset analysis demonstrated an improvement in 3-year diseasefree survival (DFS) associated with chemoradiotherapy in patients with node-positive disease (76\% vs $72 \%$ with chemotherapy) and intestinal type tumors (94\% vs $83 \%$ with chemotherapy). ${ }^{17}$ Therefore, the authors have initiated a new trial (ARTIST 2; ClinicalTrials.gov identifier: NCT01761461) focusing on patients with node-positive, D2-resected gastric cancer, which is currently active.

Preoperative chemoradiotherapy is also included in current guidelines, based on a randomized controlled trial in patients with distal esophageal and gastroesophageal cancer. In the CROSS trial, patients randomized to chemoradiotherapy followed by surgery showed an improvement in 5 -year overall survival $(47 \%)$ compared with those treated with surgery alone (34\%)..$^{20}$ It should be noted that only $24 \%$ of the patients had GEJ tumors and $23 \%$ had squamous cell carcinoma histology. Patients in this study underwent either a transthoracic resection with 2-field lymph node dissection or transhiatal resection, with gastric tube reconstruction with a cervical anastomosis, demonstrating that this study may have limited application in patients with gastric cancer. ${ }^{20}$ However, there are several phase II trials of preoperative induction chemotherapy and chemoradiotherapy that demonstrate this approach is feasible with good response rates, RO resection rates, and survival. ${ }^{21-23}$ It should be noted that there are no randomized trials of preoperative chemoradiation therapy for gastric cancer that does not involve the GEJ, and current NCCN recommendations are limited to category $2 \mathrm{~B}$ for these types of lesions.

In another phase III study, the POET trial from Germany, patients with locally advanced cancer of the lower esophagus and GEJ were randomized to either chemotherapy followed by surgery versus chemotherapy followed by chemoradiation followed by surgery. ${ }^{24}$ The study was closed early due to low accrual, with only a nonsignificant trend noted toward improved survival in the patients treated with preoperative chemoradiation therapy. However, there were significantly higher pathologic complete response rates and tumor-free lymph node rates found in the patients treated with preoperative chemoradiation.

The CRITICS trial essentially compared the MAGIC regimen of perioperative chemotherapy to preoperative chemotherapy plus an Intergroup 116 approach of postoperative chemoradiotherapy. Recently presented at the 2016 ASCO Annual Meeting, the results of this trial showed that 5-year overall survival did not differ between the 2 arms (41\% in both). ${ }^{25}$ The authors noted that only approximately $50 \%$ of patients completed the full treatment, again 
raising questions about the need to administer therapy preoperatively. Figure 1 displays the reported completion rates for the treatment arms of randomized trials comparing surgery alone versus surgery with chemotherapy or chemoradiotherapy.

\section{Surgery for Localized Gastric Cancer}

Patients medically fit for surgery with T1 lesions not amenable to EMR or ESD should be treated with upfront surgery. This practice is dependent on reliable endoscopic ultrasound, and consideration should be given to D2 lymphadenectomy due to the potential for upstaging on postoperative pathology. D1 lymphadenectomy includes the regional nodes along the lesser and greater curve and peripyloric area. D2 dissection includes D1 nodes and also all of the nodes along the left gastric artery, common hepatic artery, celiac artery, splenic hilum, and splenic artery. ${ }^{1}$

Extent of lymphadenectomy has been well studies in both Eastern and Western populations. The Eastern studies reliably justify D2 dissection as the standard of care, whereas Western studies are less clear. Historical trials of extended lymphadenectomy in Western populations include the MRC trial and the Dutch trial, ${ }^{26,27}$ which failed to show a benefit with extended lymphadenectomy, attributed to the high perioperative morbidity $(43 \%-46 \%)$ and mortality $(10 \%-14 \%)$ associated with pancreatectomy and splenectomy. ${ }^{26-29}$ The Italian IGCSG-R01 trial demonstrated a low mortality and morbidity with D2 lymphadenectomy when routine pancreatectomy and splenectomy was avoided. This trial also failed to show a difference in survival on long-term followup, which may be related to the relatively small difference in the median of retrieved lymph nodes for patients with D1 $(\mathrm{N}=25)$ and D2 $(\mathrm{N}=33)$ lymphadenectomy, the rate of contamination in the D1 group (18\%), and noncompliance in the D2 group (34\%). ${ }^{30}$ In addition, one-third of the patients had pathologic T1 lesions and more than $40 \%$ had NO disease. ${ }^{30}$

Conversely, the long-term results of the Dutch trial showed an improvement in cancer-related mortality and locoregional recurrence in the D2 group. ${ }^{31}$ In addition, on subgroup analysis of the Italian trial, a benefit for extended lymphadenectomy was identified in patients with locally advanced gastric cancer and positive lymph nodes. ${ }^{30}$ Expert centers also show a low risk associated with
D2 lymphadenectomy, and report an independent association with improved survival. ${ }^{32,33}$ Therefore, gastric cancer guidelines from Italy, Germany, Britain, ESMO/European Society of Surgical Oncology/European Society for Radiotherapy \& Oncology, and NCCN continue to recommend extended lymphadenectomy. ${ }^{1,34-37}$

The appropriate surgical approach for GEJ cancer is also defined, but requires flexibility in its application, particularly for patients with advanced tumors. Current AJCC staging classifies the pathologic stage of any patient with GEJ involvement and up to $5 \mathrm{~cm}$ of gastric involvement according to esophageal cancer criteria. ${ }^{38,39}$ Pathologic staging differs from treatment recommendations, however, because the Siewert classification is used to help determine whether to use a gastric or esophageal cancer surgical approach for tumors involving the GEJ. In short, the tumor center in a Siewert I lesion is more than $1 \mathrm{~cm}$ above the GEJ, between $1 \mathrm{~cm}$ above and $2 \mathrm{~cm}$ below the GEJ for Siewert II lesions, and more than $2 \mathrm{~cm}$ below the GEJ for a type III lesion. ${ }^{40}$ Current recommendations suggest treatment according to esophageal guidelines for a type I and II lesion and gastric guidelines for a type III lesion. ${ }^{1}$ In clinical practice, and particularly in Western populations with advanced gastroesophageal cancers that involve a considerable portion of the stomach, a combined approach with both a thoracic surgery and a surgical oncology team is helpful to obtain a margin negative resection. Recent studies support this approach for Siewert II lesions. ${ }^{33}$

\section{Summary}

There is anatomic, histologic, and surgical heterogeneity in the current trials of chemotherapy and chemoradiotherapy in gastric cancer, and it may not be appropriate to apply treatment recommendations from trials in Eastern populations to patients with gastric cancer in the United States. However, there are high-quality trials to support the current guidelines for the treatment of patients with gastric cancer that include perioperative chemotherapy, postoperative chemoradiation therapy, preoperative chemoradiation therapy, and postoperative chemotherapy. Additional research is needed to help define the optimal approach to tumors of the GEJ and also to clarify the benefit of extended lymphadenectomy. 
Badgwell

Future studies to identify which patients respond to preoperative treatment are needed to better allocate patients to the various approaches to chemotherapy and chemoradiotherapy.

\section{References}

1. Ajani JA, D'Amico TA, Almhanna $K$, et al. NCCN Clinical Practice Guidelines in Oncology: Gastric Cancer. Version 3.2016, Accessed June 12,2016 . To view the most recent version of these guidelines, visit NCCN. org.

2. Torre LA, Bray F, Siegel RL, et al. Global cancer statistics, 2012. CA Cancer J Clin 2015;65:87-108.

3. Strong VE, Song KY, Park $\mathrm{CH}$, et al. Comparison of gastric cancer survival following RO resection in the United States and Korea using an internationally validated nomogram. Ann Surg 2010;251:640-646.

4. Strong VE, Song KY, Park CH, et al. Comparison of disease-specific survival in the United States and Korea after resection for early-stage node-negative gastric carcinoma. J Surg Oncol 2013;107:634-640.

5. Strong VE, Wu AW, Selby LV, et al. Differences in gastric cancer survival between the U.S. and China. J Surg Oncol 2015;112:31-37.

6. Charalampakis N, Xiao L, Elimova E, et al. Initial standardized uptake value of positron emission tomography influences the prognosis of patients with localized gastric adenocarcinoma treated preoperatively. Oncology 2015;89:305-310.

7. Stahl A, Ott K, Weber WA, et al. FDG PET imaging of locally advanced gastric carcinomas: correlation with endoscopic and histopathological findings. Eur J Nucl Med Mol Imaging 2003;30:288-295.

8. Vallbohmer D, Holscher AH, Schneider PM, et al. [18F]fluorodeoxyglucose-positron emission tomography for the assessment of histopathologic response and prognosis after completion of neoadjuvant chemotherapy in gastric cancer. J Surg Oncol 2010;102:135-140.

9. Badgwell B, Cormier JN, Krishnan S, et al. Does neoadjuvant treatment for gastric cancer patients with positive peritoneal cytology at staging laparoscopy improve survival? Ann Surg Oncol 2008;15:2684-2691.

10. Shiozaki H, Elimova E, Slack RS, et al. Prognosis of gastric adenocarcinoma patients with various burdens of peritoneal metastases. J Surg Oncol 2016;113:29-35.

11. Badgwell B, Roy-Chowdhuri S, Chiang YJ, et al. Long-term survival in patients with metastatic gastric and gastroesophageal cancer treated with surgery. J Surg Oncol 2015;111:875-881.

12. Cunningham D, Allum WH, Stenning SP, et al. Perioperative chemotherapy versus surgery alone for resectable gastroesophageal cancer. N Engl J Med 2006;355:11-20.

13. Ychou M, Boige V, Pignon JP, et al. Perioperative chemotherapy compared with surgery alone for resectable gastroesophageal adenocarcinoma: an FNCLCC and FFCD multicenter phase III trial. J Clin Oncol 2011;29:1715-1721.

14. Diaz-Nieto R, Orti-Rodriguez R, Winslet M. Post-surgical chemotherapy versus surgery alone for resectable gastric cancer. Cochrane Database Syst Rev 2013:CD008415.

15. Earle CC, Maroun JA. Adjuvant chemotherapy after curative resection for gastric cancer in non-Asian patients: revisiting a meta-analysis of randomised trials. Eur J Cancer 1999;35:1059-1064.

16. Noh SH, Park SR, Yang HK, et al. Adjuvant capecitabine plus oxaliplatin for gastric cancer after D2 gastrectomy (CLASSIC): 5-year follow-up of an open-label, randomised phase 3 trial. Lancet Oncol 2014;15:1389-1396.

17. Park SH, Sohn TS, Lee J, et al. Phase III trial to compare adjuvant chemotherapy with capecitabine and cisplatin versus concurrent chemoradiotherapy in gastric cancer: final report of the adjuvant chemoradiotherapy in stomach tumors trial, including survival and subset analyses. J Clin Oncol 2015;33:3130-3136.

18. Macdonald JS, Smalley SR, Benedetti J, et al. Chemoradiotherapy after surgery compared with surgery alone for adenocarcinoma of the stomach or gastroesophageal junction. N Engl J Med 2001;345:725-730.

19. Smalley SR, Benedetti JK, Haller DG, et al. Updated analysis of SWOG-directed intergroup study 0116: a phase III trial of adjuvant radiochemotherapy versus observation after curative gastric cancer resection. J Clin Oncol 2012;30:2327-2333.
20. van Hagen $P$, Hulshof MC, van Lanschot JJ, et al. Preoperative chemoradiotherapy for esophageal or junctional cancer. N Engl J Med 2012;366:2074-2084.

21. Ajani JA, Mansfield PF, Janjan N, et al. Multi-institutional trial of preoperative chemoradiotherapy in patients with potentially resectable gastric carcinoma. J Clin Oncol 2004;22:2774-2780.

22. Ajani JA, Winter $K$, Okawara GS, et al. Phase II trial of preoperative chemoradiation in patients with localized gastric adenocarcinoma (RTOG 9904): quality of combined modality therapy and pathologic response. J Clin Oncol 2006;24:3953-3958.

23. Ajani JA, Mansfield PF, Crane $\mathrm{CH}$, et al. Paclitaxel-based chemoradiotherapy in localized gastric carcinoma: degree of pathologic response and not clinical parameters dictated patient outcome. J Clin Oncol 2005;23:1237-1244.

24. Stahl M, Walz MK, Stuschke M, et al. Phase III comparison of preoperative chemotherapy compared with chemoradiotherapy in patients with locally advanced adenocarcinoma of the esophagogastric junction. J Clin Oncol 2009;27:851-856.

25. Verjeij M, Jansen EP, Cats A, et al. A multicenter randomized phase III trial of neo-adjuvant chemotherapy followed by surgery and chemotherapy or by surgery and chemoradiotherapy in resectable gastric cancer: first results from the CRITICS study [abstract]. J Clin Oncol 2016;34(Suppl):Abstract 4000 .

26. Cuschieri A, Weeden S, Fielding J, et al. Patient survival after D1 and D2 resections for gastric cancer: long-term results of the MRC randomized surgical trial. Surgical Co-operative Group. Br J Cancer 1999;79:15221530 .

27. Bonenkamp JJ, Hermans J, Sasako M, et al. Extended lymph-node dissection for gastric cancer. N Engl J Med 1999;340:908-914.

28. Cuschieri A, Fayers P, Fielding J, et al. Postoperative morbidity and mortality after D1 and D2 resections for gastric cancer: preliminary results of the MRC randomised controlled surgical trial. The Surgical Cooperative Group. Lancet 1996;347:995-999.

29. Hartgrink HH, van de Velde CJ, Putter H, et al. Extended lymph node dissection for gastric cancer: who may benefit? Final results of the randomized Dutch gastric cancer group trial. J Clin Oncol 2004;22:20692077.

30. Degiuli M, Sasako M, Ponti A, et al. Randomized clinical trial comparing survival after D1 or D2 gastrectomy for gastric cancer. Br J Surg 2014;101:23-31.

31. Songun I, Putter H, Kranenbarg EM, et al. Surgical treatment of gastric cancer: 15-year follow-up results of the randomised nationwide Dutch D1D2 trial. Lancet Oncol 2010;11:439-449.

32. Badgwell B, Ajani J, Blum M, et al. Postoperative morbidity and mortality rates are not increased for patients with gastric and gastroesophageal cancer who undergo preoperative chemoradiation therapy. Ann Surg Oncol 2016;23:156-162.

33. Kneuertz PJ, Hofstetter WL, Chiang YJ, et al. Long-term survival in patients with gastroesophageal junction cancer treated with preoperative therapy: do thoracic and abdominal approaches differ? Ann Surg Oncol 2016;23:626-632.

34. De Manzoni G, Baiocchi GL, Framarini M, et al. The SIC-GIRCG 2013 Consensus Conference on gastric cancer. Updates Surg 2014;66:1-6.

35. Allum WH, Blazeby JM, Griffin SM, et al. Guidelines for the management of oesophageal and gastric cancer. Gut 2011;60:1449-1472.

36. Waddell T, Verheij M, Allum W, et al. Gastric cancer: ESMO-ESSO ESTRO clinical practice guidelines for diagnosis, treatment and follow-up. Eur J Surg Oncol 2014;40:584-591.

37. Moehler M, Al-Batran SE, Andus T, et al. [German S3-guideline "Diagnosis and treatment of esophagogastric cancer"]. Z Gastroenterol 2011;49:461-531 [in German].

38. Stomach cancer. In: Edge S, Byrd D, Compton C, et al, eds. AJCC Cancer Staging Manual, 7th ed. New York, NY: Springer; 2010:117-126.

39. Esophagus and esophagogastric junction. In: Edge S, Byrd D, Compton C, et al, eds. AJCC Cancer Staging Manual, 7th ed. New York, NY: Springer; 2010:103-111.

40. Siewert JR, Stein HJ. Classification of adenocarcinoma of the oesophagogastric junction. Br J Surg 1998;85:1457-1459.

41. Sasako M, Sakuramoto S, Katai H, et al. Five-year outcomes of a randomized phase III trial comparing adjuvant chemotherapy with S-1 versus surgery alone in stage II or III gastric cancer. J Clin Oncol 2011;29:4387-4393. 
Multimodality Therapy for Gastric Cancer

\section{Instructions for Completion}

To participate in this journal CE activity: 1) review the learning objectives and author disclosures; 2 ) study the education content; 3 ) take the posttest with a $66 \%$ minimum passing score and complete the evaluation at http://education.nccn.org/ node/79642; and 4) view/print certificate. After reading the article, you should be able to answer the following multiple- choice questions. Credit cannot be obtained for tests completed on paper. You must be a registered user on NCCN.org. If you are not registered on NCCN.org, click on "New Member? Sign up here" link on the left hand side of the Web site to register. Only one answer is correct for each question. Once you successfully answer all posttest questions you will be able to view and/or print your certificate. Software requirements: Internet

\section{Posttest Questions}

1. True or False: Perioperative chemotherapy is associated improved overall survival compared with surgery alone in patients with potentially resectable gastric cancer.

2. The current recommendations for postoperative chemoradiotherapy are primarily based on the trial, although preoperative chemoradiotherapy is based primarily on the trial.

a. CLASSIC; ARTIST

b. Intergroup 116; CROSS

c. CRITICS; ARTIST 2

4. True or False: Patients medically fit for surgery with TI lesions, not amenable to EMR or ESD, should be treated with upfront surgery. 